\title{
Functional network interactions at rest underlie individual differences in memory ability
}

\author{
Mariët van Buuren, ${ }^{1,2,4}$ Isabella C. Wagner, ${ }^{2,3,4}$ and Guillén Fernández ${ }^{2}$ \\ ${ }^{1}$ Department of Clinical, Neuro and Developmental Psychology, Faculty of Behavioral and Movement Sciences, Institute for Brain \\ and Behavior Amsterdam, Vrije Universiteit Amsterdam, 1081 BT Amsterdam, The Netherlands; ${ }^{2}$ Donders Institute for Brain, Cognition \\ and Behaviour, Radboud University Nijmegen Medical Centre, 6500 HB, Nijmegen, The Netherlands; ${ }^{3}$ Social, Cognitive and Affective \\ Neuroscience Unit, Department of Basic Psychological Research and Research Methods, Faculty of Psychology, University of Vienna, \\ 1010 Vienna, Austria
}

\begin{abstract}
Intrinsic network interactions may underlie individual differences in the ability to remember. The default mode network (DMN) comprises subnetworks implicated in memory, and interactions between the DMN and frontoparietal network (FPN) were shown to support mnemonic processing. However, it is unclear if such interactions during resting-state predict episodic memory ability. We investigated whether intrinsic network interactions within and between the DMN and FPN are related to individual differences in memory performance. Resting-state activity was measured using functional MRI in healthy young adults followed by a memory test for object-location associations that were studied $3 \mathrm{~d}$ earlier. We identified two subnetworks within the DMN, the main-DMN and the medial temporal lobe, retrosplenial cortex (MTL_RSC)-DMN. Further, we found regions forming the FPN. Memory performance was associated with lower connectivity within the MTL_RSC-DMN, and stronger connectivity between the main-DMN and FPN. Exploratory whole-brain analysis revealed stronger MTL connectivity with the left posterior parietal cortex that was related to better memory performance. Furthermore, we found increased task-evoked activation during successful retrieval within the main-DMN and FPN, but not within the MTL_RSC-DMN. In sum, lower intrinsic connectivity within the MTL_RSC-DMN, combined with stronger connectivity between the main-DMN and FPN, explain individual differences in memory ability.
\end{abstract}

[Supplemental material is available for this article.]

We all differ in our ability to learn and remember information. Not only variance in task-evoked brain responses, but also functional interactions between large-scale brain networks during rest may describe brain properties that underlie trait differences in our ability to learn (Gerraty et al. 2014) and memorize (Wang et al. 2010; Salami et al. 2014; Spreng et al. 2018). The default mode network $(\mathrm{DMN})$ was originally defined as a set of regions showing increased activation during rest compared to goal-directed, complex tasks (Shulman et al. 1997; Raichle et al. 2001). Key regions of the DMN include the dorsal and ventral medial prefrontal cortex, the posterior cingulate cortex, retrosplenial cortex (RSC), lateral temporal lobes, and the posterior inferior parietal lobes (including the angular gyri) (Gusnard et al. 2001; Raichle et al. 2001; Greicius et al. 2003; Fox et al. 2005; Buckner et al. 2008). Several studies have shown that the DMN consists of interacting subsystems that are involved in different aspects of internally focused cognition, including self-referential processing and memory processing (Buckner et al. 2008; Kumaran et al. 2009; van Buuren et al. 2010; Andrews-Hanna et al. 2010, 2014a,b; Kaboodvand et al. 2018; Staffaroni et al. 2018), although multiple fractionations of the network were proposed. An analysis of intrinsic activity of the DMN by Andrews-Hanna et al. (2010) described a core subsystem (the core-DMN), comprising the anterior medial prefrontal and posterior cingulate cortices serving as hubs, that interacted with two other subsystems; a dorsal medial and a medial temporal lobe (MTL) subsystem (Andrews-Hanna et al. 2010). Other studies have reported a

${ }^{4}$ These authors contributed equally to this work. Corresponding author: vanbuurenmariet@gmail.com Article is online at http://www.learnmem.org/cgi/doi/10.1101/lm.048199.118. division of the cortical DMN regions into an anterior and posterior subnetwork (Damoiseaux et al. 2008), and an MTL-subsystem (Salami et al. 2014; Kaboodvand et al. 2018; Staffaroni et al. 2018). Regions of the MTL-subsystem, as well as cortical medial posterior regions, the angular gyrus, and the anterior medial prefrontal cortex, appear to be involved in autobiographical and episodic memory retrieval (Andrews-Hanna et al. 2010, 2014a,b; Daselaar et al. 2004, 2009; Vilberg and Rugg 2008; Sestieri et al. 2011; Huijbers et al. 2012; Benoit and Schacter 2015). Moreover, a recent study probing age-related changes reported connectivity changes within a cortical DMN network and between anterior and posterior DMN subnetworks to predict changes in memory performance (Staffaroni et al. 2018), highlighting the importance of these subnetworks in mnemonic processing.

Not only interactions within the DMN but also interactions between the DMN and other large-scale networks (Vatansever et al. 2015), in particular the frontoparietal network (FPN, or executive control network), appear to support cognitive abilities (Spreng et al. 2010; Cocchi et al. 2013). The FPN comprises the lateral prefrontal cortex, dorsal anterior cingulate cortex, precuneus, anterior inferior parietal lobule, and the inferior temporal cortex (Vincent et al. 2008; Yeo et al. 2011; Spreng et al. 2013), and is implicated in cognitive control (Cocchi et al. 2013; Cole et al. 2013), attention (Markett et al. 2014), and memory processes (Borst and Anderson

(C) 2019 van Buuren et al. This article is distributed exclusively by Cold Spring Harbor Laboratory Press for the first 12 months after the full-issue publication date (see http://learnmem.cshlp.org/site/misc/terms.xhtml). After 12 months, it is available under a Creative Commons License (Attribution-NonCommercial 4.0 International), as described at http://creativecommons.org/licenses/by-nc/ $4.0 \%$. 
2013). A study segregating the brain's resting-state activity into an intrinsic and an extrinsic system (related to internal and external processing, respectively) (Doucet et al. 2011) reported that, although the DMN and FPN formed distinct networks, both the DMN and FPN were assigned to the same, intrinsic system. This suggests that the DMN and FPN are cooperative rather than competitive networks (Doucet et al. 2011; see also, Andrews-Hanna et al. 2014b). Moreover, task-based interactions between the DMN and FPN were found to contribute to successful (King et al. 2015) and more rapid memory retrieval (Fornito et al. 2012), as well as memory search (Kragel and Polyn 2015); thus, revealing cooperative interplay between these networks in support of mnemonic processing. Additionally, a recent study reported increased intrinsic coupling between the lateral prefrontal cortex and DMN to predict more semanticized autobiographical memories in older (but not younger) adults (Spreng et al. 2018). However, it is unclear whether intrinsic interactions within and between the DMN and FPN are related to episodic mnemonic processing in young adults, and whether they underlie individual differences in the ability to remember.

Our goal was to investigate whether intrinsic network interactions within and between the DMN and FPN are related to memory ability, or more specifically, to the individual variability in episodic memory performance. To this aim, we measured brain activity during resting-state using functional MRI in 24 young adults, followed by a memory test for associations that were studied $3 \mathrm{~d}$ earlier (Fig. 1A). We used a spatial associative memory task in which participants studied and recalled associations between objects and locations to minimize the potential influence of self-referential and social processing on task performance and activation in the DMN (Fig. 1B,C; Meyer et al. 2018). Since we were interested in more ecologically valid, longer-term memories, memory performance was defined as the number of associations correctly recalled immediately after learning as well as at the delayed test after $3 \mathrm{~d}$. Additionally, brain activity changes during the delayed test were assessed to validate engagement of the DMN and FPN in actual memory retrieval.

We hypothesized that trait-like interactions within the DMN and between the DMN and the FPN would explain individual differences in memory performance. To test this hypothesis, restingstate connectivity between regions of the DMN and FPN was analyzed and subsystems were identified using hierarchical cluster analysis. Next, the individual average connectivity scores within and between the resulting subsystems were submitted into a stepwise multiple regression analysis with memory performance as a dependent variable. We predicted stronger connectivity within an MTL-subsystem of the DMN, and of both this MTL-subsystem and the core-DMN with the FPN to be associated with better memory performance. Additionally, we expected the MTL-subsystem of the DMN, the core-DMN, as well as the FPN, to reveal increased activation during correct relative to incorrect memory retrieval.

\section{Results}

\section{Memory performance}

The goal of this study was to investigate whether network interactions during resting-state are associated with individual variation in associative memory performance. Successful memory performance was significantly above chance level (1 correct association/ 64 possible locations $=1.60 \%$ ). On average, participants correctly recalled $\sim 44 \%$ of all learned associations (mean \pm SE: $28.17 \pm 2.66$ correct associations, $t_{(23)}=10.56, P<0.0005$, test-value $=0.016$ ) . About $45 \%$ of the learned associations were recalled incorrectly (mean \pm SE: $29.17 \pm 2.74$ incorrect associations), and participants failed to respond to very few trials only (mean \pm SE: $4.04 \pm 0.68$ misses). Importantly, successful memory performance varied across par-

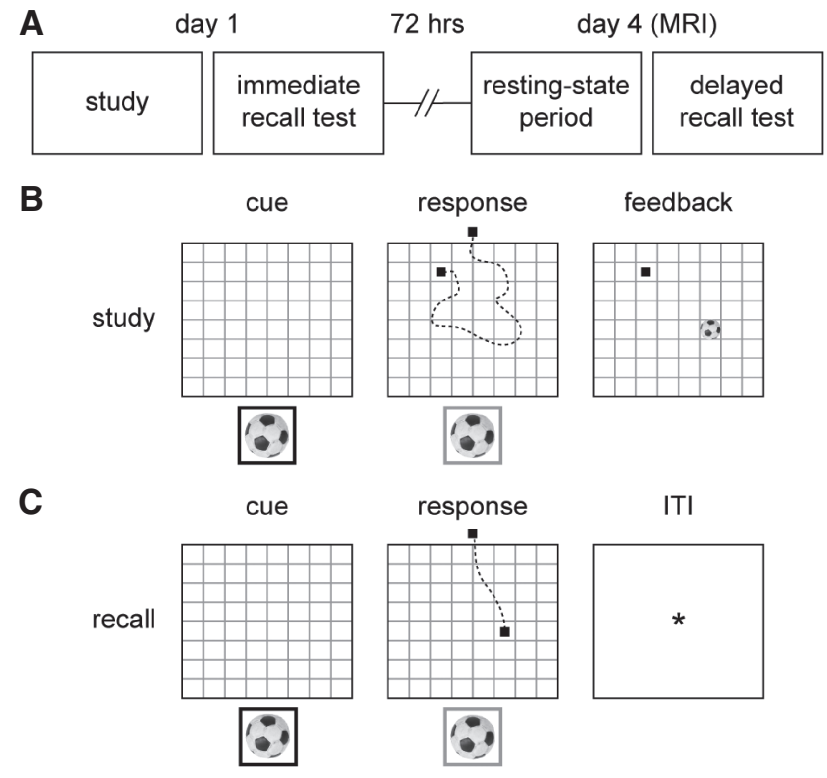

Figure 1. Experimental design $(A)$ Experimental procedure. On the first day, participants performed an object-location association memory task on a computer in a behavioral laboratory. Seventy-two hours later, participants returned for a session in the MR scanner. Brain activity was measured using functional MRI during a resting-state period and subsequently while the participants performed the delayed recall test of the memory task. (B) At the start of the study phase, all 64 objects were displayed at their correct locations within the grid for $1.5 \mathrm{~min}$. After this, study trials were presented. A trial started with the presentation of a cue (i.e., one of the objects) in a red frame (here in black) below an empty grid. After $3 \mathrm{sec}$, the frame turned green (here in gray) and a black squared cursor appeared randomly at one of the four sides of the grid. The subject was then required to indicate the correct object-location association within $2 \mathrm{sec}$ (example of cursor trajectory is marked as dashed line). After responding, feedback was presented on the screen for $3 \mathrm{sec}$ plus the remaining response period. If the given response was incorrect (as illustrated), the cursor turned red (here in black) and the object was displayed at the correct location. (C) During the recall test, a trial again started with the presentation of a cue followed by a response period of $2.5 \mathrm{sec}$ (example of cursor trajectory is marked as dashed line). No feedback was provided. The next trial started after a variable inter-trial-interval (ITI; mean $=5 \mathrm{sec}$, range $=2.5-7 \mathrm{sec}$ ). Figure is modified from a previous publication (Wagner et al. 2017).

ticipants ranging from six to 49 associations and thus showed sufficient variance for testing individual differences in memory ability.

\section{Resting-state functional connectivity}

\section{Connectivity and network identification}

Regions of interest (ROIs) were defined by $8-\mathrm{mm}$ spheres centered on the 22 selected coordinates within the DMN and FPN based on the literature (see Table 1; Andrews-Hanna et al. 2010; Yeo et al. 2011). To define subnetworks within the DMN (and possibly within the FPN), and to identify regions that changed network alignment (i.e., regions that were originally selected as being part of the DMN but that were then identified as being part of the FPN by the cluster analysis and vice versa), we first calculated functional resting-state connectivity between all 22 ROIs and then averaged connectivity across participants. The average connectivity matrix is shown in Figure 2A. Next, we performed a hierarchical cluster analysis to define subnetworks based on this mean connectivity profile. As depicted in Figure 2B, this analysis divided the 22 ROIs into four clusters (threshold of $70 \%$ of the maximum linkage): two clusters within the DMN, one cluster forming the FPN, 
Table 1. Coordinates of regions of interest

\begin{tabular}{llrrr}
\hline & & \multicolumn{2}{l}{ MNI coordinates } \\
\cline { 3 - 5 } Brain region (abbreviation) & & \multicolumn{2}{c}{$\boldsymbol{y}$} & $\boldsymbol{z}$ \\
\hline DMN & & & & \\
Anterior medial prefrontal cortex & (aMPFC) & -6 & 52 & -2 \\
Dorsal medial prefrontal cortex & (dMPFC) & 0 & 52 & 26 \\
Ventral medial prefrontal cortex & (VMPFC) & 0 & 26 & -18 \\
Posterior cingulate cortex & (PCC) & -8 & -56 & 26 \\
Left retrosplenial cortex & (IRSC) & -14 & -52 & 8 \\
Right retrosplenial cortex & (rRSC) & 14 & -52 & 8 \\
Left temporoparietal junction & (ITPJ) & -54 & -54 & 28 \\
Right temporoparietal junction & (rTPJ) & 54 & -54 & 28 \\
Left posterior inferior parietal lobule & (IpIPL) & -44 & -74 & 32 \\
Right posterior inferior parietal lobule & (rpIPL) & 44 & -74 & 32 \\
Left lateral temporal cortex & (ILTC) & -60 & -24 & -18 \\
Right lateral temporal cortex & (rLTC) & 60 & -24 & -18 \\
Left medial temporal lobe & (IMTL) & -25 & -32 & -18 \\
Right medial temporal lobe & (rMTL) & 25 & -32 & -18 \\
FPN & & & \\
Dorsal anterior cingulate cortex & (dACC) & -5 & 22 & 47 \\
Precuneus & (Prec) & -4 & -76 & 45 \\
Left lateral prefrontal cortex & (ILPFC) & -40 & 50 & 7 \\
Right lateral prefrontal cortex & (rLPFC) & 40 & 50 & 7 \\
Left inferior temporal cortex & (IITC) & -57 & -54 & -9 \\
Right inferior temporal cortex & (rITC) & 57 & -54 & -9 \\
Left anterior inferior parietal lobule & (lalPL) & -43 & -50 & 46 \\
Right anterior inferior parietal lobule & (ralPL) & 43 & -50 & 46 \\
\hline & & & &
\end{tabular}

MNI coordinates of the center of the ROI with abbreviation in brackets. Selection of the regions is based on current literature (Andrews-Hanna et al. 2010; Yeo et al. 2011) and mirrored coordinates in the right hemisphere are included for lateral regions $(x>-10)$. I, left; $r$, right.

and one cluster consisting of the precuneus and the right posterior inferior parietal lobule that were originally part of the FPN and DMN, respectively, when selecting the ROIs based on previous literature. Since the latter cluster comprised only two regions that each were originally affiliated with distinct networks, this cluster was not included in the remaining analyses. The two clusters of the DMN consisted of (1) the main-DMN, that included the ventral, anterior and dorsal medial prefrontal cortex, posterior cingulate cortex, left posterior inferior parietal lobule, bilateral temporoparietal junction, and the bilateral lateral temporal cortex, and (2) the MTL_RSC-DMN comprising the bilateral MTL and RSC (Fig. 2C). We refer to this cluster as the "MTL_RSC-DMN" to make it explicit that no regions except the MTL and RSC were included. The FPN consisted of the dorsal anterior cingulate cortex, bilateral lateral prefrontal cortex, inferior temporal cortex, and the anterior inferior parietal lobule (Fig. 2C), all part of the FPN when defining the ROIs (see Materials and Methods, Definition of regions of interest). Changing the clustering threshold to correspond to a maximum number of 5 or 6 clusters did not change the partition of the DMN.

Next, we assessed the strength of the within- and betweennetwork connectivity as defined by the hierarchical cluster analysis. To avoid circularity, we performed this verification using resting-state data of an independent group of 25 participants. Connectivity (Fisher's $z$-scores, $\mathrm{Fz}$ ) within the three networks was significantly above zero (main-DMN: mean $\mathrm{Fz}=0.46, t_{(24)}=27.22$, $P<0.0005$; MTL_RSC-DMN: mean Fz $=0.54, t_{(24)}=22.85, \quad P<$ 0.0005; FPN: mean $\left.\mathrm{Fz}=0.41, t_{(24)}=19.40, P<0.0005\right)$, and the average within-network connectivity was stronger compared to the average between-network connectivity (mean Fz: 0.47 and 0.10, respectively, $t_{(24)}=24.37, P<0.0005$ ). In addition, connectivity between the two networks of the DMN was stronger than the average connectivity of these networks with the FPN (mean Fz: 0.21 and 0.05 , respectively, $\left.t_{(23)}=5.99, P<0.0005\right)$.
In sum, the cluster analysis identified two networks within the DMN, namely, the main-DMN and the MTL_RSC-DMN, and a network of regions forming the FPN. As expected, connectivity within these three networks was higher as compared to connectivity between the networks. Additionally, a fourth cluster consisting of the precuneus and the right posterior inferior parietal lobule emerged. Contrary to what we expected, the posterior cingulate and anterior medial prefrontal cortex did not form a separate cluster (i.e., the core-DMN), but were aligned with the main-DMN network.

\section{Network connectivity is associated with memory performance}

We hypothesized that interactions within the DMN and between the DMN and the FPN would be related to individual memory ability. To this aim, the individual average connectivity scores within the MTL_RSC-DMN, the main-DMN, and FPN, and between these three networks were submitted to a stepwise multiple regression analysis with memory performance as a dependent variable.

As a first step, connectivity within the MTL_RSC-DMN was entered into the regression equation and significantly predicted memory performance $\left(\beta=-0.53, t_{(22)}=-2.89, P=0.008\right)$, explaining $27.5 \%$ of the variance in memory performance $\left(F_{(1,22)}=8.35\right.$, $P=0.008)$. Next, connectivity between the main-DMN and FPN was entered to the equation, resulting in an increase in explained variance of memory performance by $23.7 \%\left(F_{(1,21)}=10.21, P=\right.$ 0.004 , Durbin-Watson $=2.48$ ). In this final model, $51.2 \%$ of the variability in memory performance was explained $\left(R^{2}=0.51\right.$, adjusted $\left.R^{2}=0.47, F_{(2,21)}=11.02, P=0.001\right)$. Collinearity statistics (tolerance) was $\sim 0.55$ for all independent variables (included and excluded in the model), and the assumption of homoscedasticity and normality of the residuals was met. Specifically, memory performance was positively associated with lower connectivity within the MTL_RSC-DMN $\left(\beta=-0.50, t_{(21)}=-3.27, P=0.004\right)$ (Fig. $\left.3 \mathrm{~A}\right)$, in combination with stronger connectivity between the mainDMN and FPN $\left(\beta=0.49, t_{(21)}=3.19, p=0.004\right)$ (Fig. 3B). Remaining within- and between-network interactions did not enter the model and thus did not significantly contribute to explaining variance in successful memory performance (main-DMN: $t_{(21)}=0.83, P=0.42$; FPN: $t_{(21)}=-0.40, P=0.69$; main-DMN by MTL_RSC-DMN: $t_{(21)}=$ $-0.036, P=0.97$; MTL_RSC-DMN by FPN: $\left.t_{(21)}=1.00, P=0.33\right)$. Thus, the resulting regression equation for predicting memory performance was

Predicted memory performance $=50.33-\left(51.74 \mathrm{x}_{\text {MTL_RSC }- \text { DMN }}\right)$

$$
+\left(83.48 \mathrm{x}_{\text {main }-\mathrm{DMN} \times \mathrm{FPN}}\right) \text {. }
$$

To further probe the reported association between lower within MTL_RSC-DMN connectivity and memory performance, we performed two exploratory whole-brain connectivity analyses with the left and right MTL regions combined as one seed region, and the left and right retrosplenial cortex combined as a second seed region. Successful memory performance was entered as a covariate at the group level. More specifically, we calculated correlation coefficients (Pearson's $r$ ) between the average time course of each seed region with all other voxels in the brain and converted resulting correlations to $\mathrm{Fz}$. These Fz-weighed images were submitted to a one-sample $t$-test with successful memory performance added as a covariate. Significance was assessed using a cluster-defining threshold of $P<0.001$ and a $P<0.05$ FWE-corrected critical cluster size of 29 voxels. Significant clusters were labeled using the Laboratory of Neuro Imaging (LONI) Probabilistic Brain Atlas (Shattuck et al. 2008). We found connectivity between the bilateral MTL and left supramarginal gyrus, extending into the angular gyrus, to be positively related to memory performance (peak coordinate 
A

ARSC

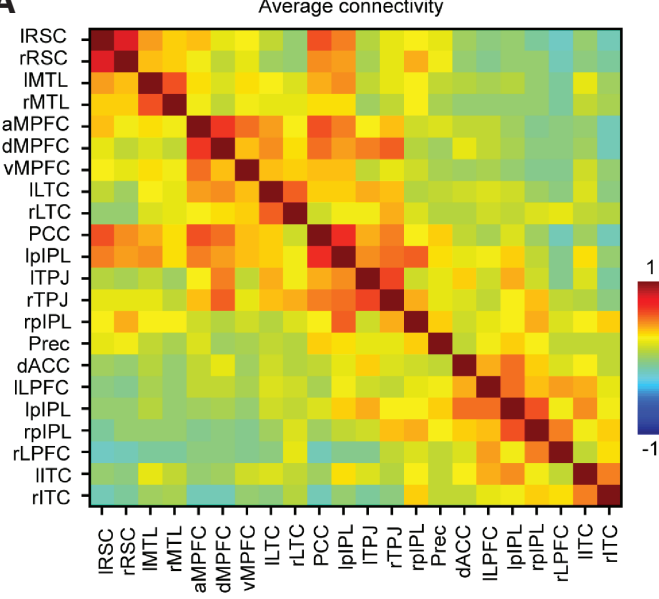

B

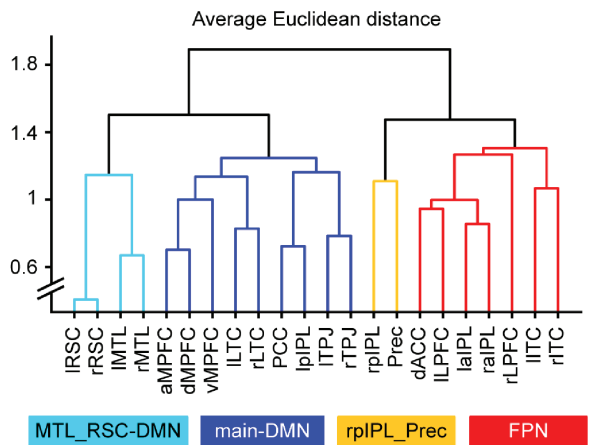

C
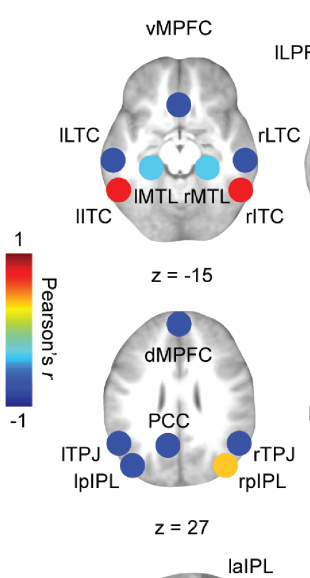

$z=27$

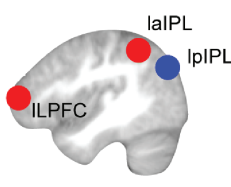

$x=-58$

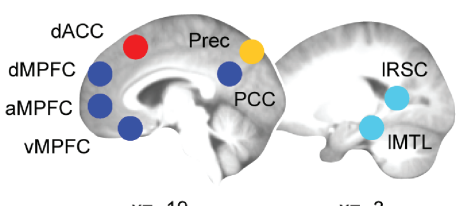

$x=-19$

Figure 2. Average connectivity and network identification. (A) Average connectivity values (Pearson's $r$ ) over subjects of all ROI-to-ROI correlations. Warmer colors indicate higher connectivity. (B) Dendrogram of the hierarchical cluster analysis revealing the four networks; MTL_RSC-DMN (cyan), main-DMN (blue), FPN (red), and a fourth cluster (orange). (C) Colored regions of each network, MTL_RSC-DMN (cyan), main-DMN (blue), FPN (red), and a fourth cluster (orange), are schematically indicated by spheres and overlaid on a mean anatomical image. Explanations of the abbreviations of the regions are found in Table 1.

of local maxima, $Z$-value, and cluster size: $x, y, z=-52,-49,49 ; Z=$ $3.71 ; 29$ voxels; see Fig. 3C). No significant interaction between whole-brain connectivity of the RSC seed with memory performance was found.

In sum, memory performance was predicted by lower connectivity within the MTL_RSC-DMN network, combined with stronger connectivity between the main-DMN and FPN at rest. Together, these interactions accounted for $51.2 \%$ of the individual variability in memory performance. Moreover, exploratory wholebrain analysis revealed stronger connectivity between the bilateral MTL with the left angular gyrus to be associated with better memory performance.

To demonstrate the stability of our results, we performed four additional control analyses (see Supplemental Materials and Methods and Supplemental Results). First, incorporating global signal regression instead of partial global signal regression again revealed two subnetworks within the DMN (the main-DMN and MTL_RSC-DMN), one set of regions forming the FPN, and a cluster comprising the precuneus and right posterior inferior parietal lobule. Furthermore, the resulting prediction model of individual differences in memory performance remained similar. Second, cluster analysis on resting-state data of an independent group of participants revealed the same four subnetworks when a clustering threshold of four was applied, indicating that this network architecture was reliable. Third, interactions within and between two control networks, the auditory and visual network (see Supplemental

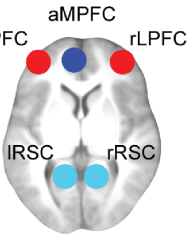

$z=3$

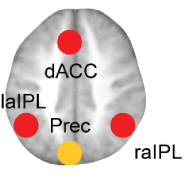

$z=43$

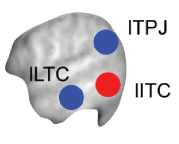

Table S1), did not predict memory performance, suggesting that the network interactions within the MTL_RSC-DMN and between the main-DMN and FPN specifically are associated with individual memory ability. Fourth, the results of the stepwise multiple regression analyses were confirmed with a Lasso approach.

\section{Task-induced signal changes during delayed memory retrieval}

To verify the involvement of the mainDMN, MTL_RSC-DMN, and the FPN in memory retrieval, we analyzed signal changes within these networks during correct compared to incorrect memory retrieval following resting-state fMRI. A repeated-measures ANOVA with network (main-DMN, MTL_RSC-DMN, FPN) and memory (correct, incorrect) as withinsubject factors revealed main effects of memory and network $\left(F_{(1,23)}=9.31, P=\right.$ 0.006 and $F_{(2,46)}=124.87, \quad P<0.0005$, respectively), together with a network by memory interaction $\left(F_{(2,46)}=8.67, P=\right.$ 0.001 ), suggesting that the three networks showed differential activation profiles related to mnemonic processing. Posthoc $t$-tests, comparing correct to incorrect memory retrieval within each network, showed increased activation of the main-DMN $\left(t_{(23)}=4.71, P<0.0005\right)$ and the FPN $\left(t_{(23)}=2.36, P=0.027\right)$ in correct memory retrieval, but no significant differential activation in the MTL_RSCDMN $\quad\left(t_{(23)}=0.39, P=0.704\right) \quad$ (Fig. 4$)$. These findings suggest that the mainDMN and FPN are related to successful retrieval processes. Unexpectedly, the MTL_RSC-DMN did not show increased brain activation related to successful memory retrieval.

To further investigate the lack of differential activation within the MTL_RSC-DMN related to memory retrieval, we probed whether the amount of accurate spatial information available during incorrect trials influenced the level of activation within the MTL_RSC-DMN. To this aim, we repeated the analysis with distance to the correct location included as a parametric modulator of the incorrect trials (see Supplemental Materials and Methods). As above, this analysis revealed no significant differential retrievalrelated activation in the MTL_RSC-DMN $\left(t_{(23)}=0.06, P=0.96\right)$, nor a significant effect of distance on MTL_RSC-DMN activity during incorrect trials $\left(t_{(23)}=0.80, P=0.435\right)$.

\section{Discussion}

We examined whether intrinsic functional network interactions underlie memory ability, and tested the hypothesis that interactions within the DMN, and between the DMN and the FPN, are related to individual differences in memory performance. Cluster analysis identified two networks within the DMN, the main-DMN and the MTL_RSC-DMN, as well as a set of regions forming the FPN. Contrary to our expectations, lower connectivity within the MTL_RSC-DMN, combined with stronger connectivity between the main-DMN and FPN, were related to better individual 
A

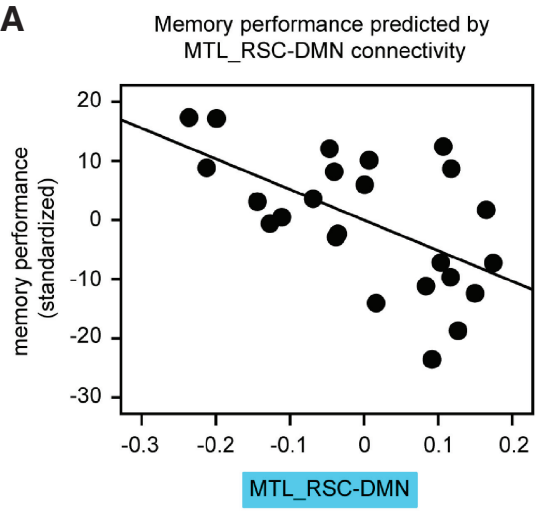

within-network connectivity (standardized)
B

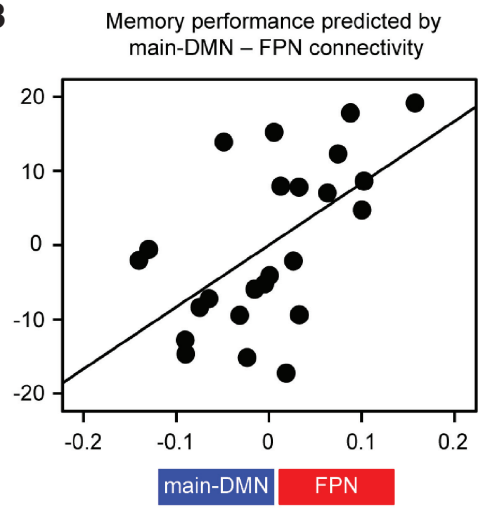

between-network connectivity (standardized)
C

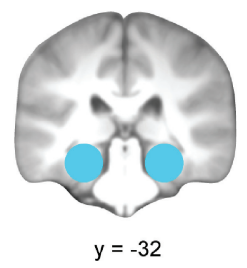

MTL connectivity covariate memory performance

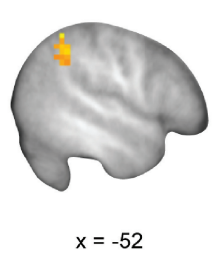

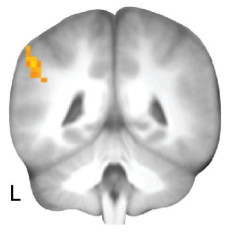

$y=-46$

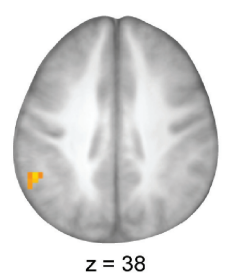

$z=38$

Figure 3. Network interactions predicting memory performance. $(A)$ Lower connectivity within the MTL_RSC-DMN, in combination with (B) stronger connectivity between the main-DMN and FPN together predict memory performance. Memory performance and connectivity values are standardized (z-scores). Panel $A$ shows the relationship between memory and MTL_RSC-DMN connectivity after removing the linear effects of main-DMN-FPN connectivity. Panel $B$ shows the relationship between memory and main-DMN-FPN connectivity after removing the linear effects of MTL_RSC-DMN connectivity. (C) Stronger connectivity between the left and right MTL with the left posterior parietal cortex at rest was associated with better memory performance. Cluster-defining threshold of $P<0.001$ and a $P<$ 0.05 FWE-corrected critical cluster size of 29 voxels. Left and right MTL are schematically indicated by spheres in cyan. MTL regions and results are overlaid on a mean anatomical image.

memory ability. In line with our connectivity findings, analyses of task-related activity revealed increased activation within the main-DMN and FPN when comparing successful to unsuccessful retrieval. However, no such differential activation was found within the MTL_RSC-DMN. To the best of our knowledge, our study is the first to show that resting-state interactions between the DMN and the FPN are associated with individual differences in episodic memory ability in young adults.

We identified two networks within the DMN and one set of regions forming the FPN. These three networks largely overlap with previous studies on the fractionation of the DMN and studies on the FPN (Vincent et al. 2008; Andrews-Hanna et al. 2010, 2014b; Spreng et al. 2010; Staffaroni et al. 2018). However, we did not find decomposition of the cortical DMN regions into an anterior and posterior component (Damoiseaux et al. 2008; Staffaroni et al. 2018), nor did we find a separate network comprising the core regions of the DMN (the posterior cingulate and anterior medial prefrontal cortex) (Andrews-Hanna et al. 2010). Instead, the cluster analysis yielded two DMN subnetworks, the main-DMN and the MTL_RSC-DMN. All cortical DMN regions except for the right posterior inferior parietal lobule were included in the main-DMN. The MTL_RSC-DMN comprised the bilateral medial temporal lobe and RSC, comparable to the MTL-DMN reported by Staffaroni and colleagues (Staffaroni et al. 2018; but see Andrews-Hanna et al. 2010 for an MTL-subsystem including the ventral medial prefrontal cortex and the posterior inferior parietal lobule). All regions of the FPN, except for the precuneus, formed the FPN cluster. Unexpectedly, the precuneus formed a separate cluster together with the right posterior inferior parietal lobule. Although in the 17-network parcellation of the cerebral cortex of Yeo et al. (2011), the precuneus was also reported as a separate network (together with a posterior cingulate cortex node), we did expect the right posterior inferior parietal lobule to be included in a DMN subnetwork. The differences in cluster formation between our study and previous findings may be due to differences in region selection as well as clustering approach which impact network formation (Arslan et al. 2018). Our region selection was based on two previous studies (Andrews-Hanna et al. 2010; Yeo et al. 2011). We excluded regions with low signal-to-noise ratio and included both left and right lateralized regions (instead of only left lateralized regions, as in Andrews-Hanna et al. 2010, 2014b), since we had no predictions for specific laterality effects (Bellana et al. 2016). Moreover, because we were interested in the interplay between the two overarching networks as well as their subnetworks, cluster analysis was performed on both the DMN and FPN regions together, instead of on whole-brain connectivity patterns or on each network separately. Importantly, aside from the precuneus and the right posterior inferior parietal lobule, none of the other regions changed their network alignment (i.e., regions that were originally selected as being part of the DMN but that were identified as being part of the FPN by the cluster analysis and vice versa). Moreover, repetition of the cluster analysis on resting-state data of an independent sample identified similar clusters of the DMN and FPN. Altogether, this suggests that although the exact decomposition of the DMN in subnetworks may differ depending on region selection and cluster analysis approach, network alignment and clustering were robust.

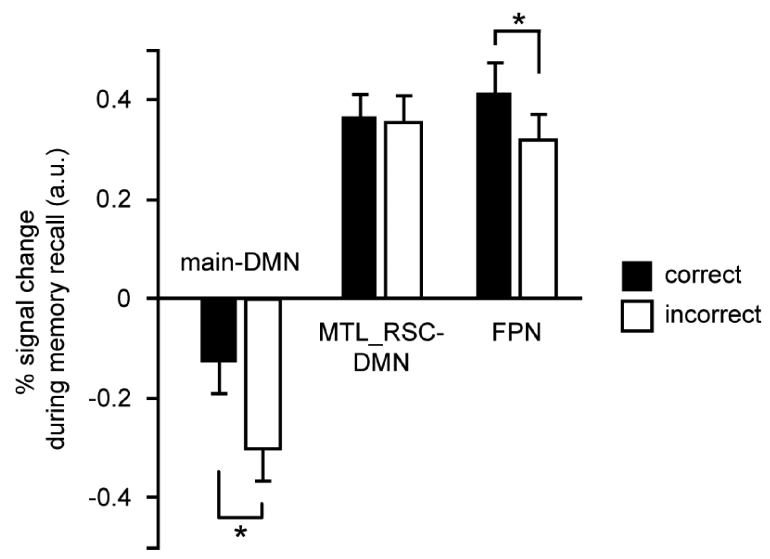

Figure 4. Activity within the network during recall. Mean percentage of signal change per network during correct relative to incorrect recall within each of the networks. Error bars represent standard error of mean. $\left(^{*}\right) P<$ 0.0005 . 
We predicted stronger connectivity within the MTL-subsystem of the DMN (i.e., the MTL_RSC-DMN), as well as between both this MTL-subsystem and the core-DMN with the FPN to be associated with better memory performance. In contrast to this, we revealed that lower within MTL_RSC-DMN connectivity and stronger connectivity between the main-DMN and FPN explained individual memory ability. In line with our finding of lower MTL_RSC-DMN connectivity associated with better memory, Salami et al. (2014) showed enhanced resting-state connectivity within a hippocampal-DMN subsystem that was positively associated with age and negatively related to memory performance. This enhanced connectivity was suggestive for more isolated hippocampal-DMN functioning and the inability to flexibly recruit this region together with other memory-related regions during memory processing (Salami et al. 2014). This hypothesis is partly supported by studies reporting a positive relationship between memory performance and hippocampal connectivity with the posterior medial cortex (Wang et al. 2010; La Joie et al. 2014), although these studies focused on hippocampal connectivity and not connectivity of an MTL-system of the DMN. Additionally, a study targeting individual differences in autobiographic episodic memory revealed stronger coupling of a parahippocampal region (similar to our MTL region included in the MTL_RSC-DMN) with parietal and occipital regions related to episodic-based remembering (Sheldon et al. 2016). Similarly, our exploratory whole-brain analysis showed that stronger MTL connectivity with the left posterior parietal cortex was associated with better memory performance. Together, these studies and our findings suggest stronger MTL coupling with regions outside the MTL_RSC-DMN system (as defined in the present study) is positively related to memory performance. However, a recent study in elderly participants did not find connectivity of the MTL-DMN to cortical DMN networks in relation to memory performance (Staffaroni et al. 2018). Furthermore, another study in elderly participants that partitioned the DMN into five subnetworks, including a separate MTL-DMN component and a component comprising the RSC, reported that stronger instead of weaker MTL-RSC connectivity was associated with better episodic memory. These differences in findings could possibly be explained by yet unknown differences in, for example, task design and stimuli used, or by differences in age of the participants. Indeed, a longitudinal study revealed changes in hippocampal-cortical connectivity that were differently associated with memory changes across young and older adults (Fjell et al. 2016). Furthermore, studies reported age-related reductions in connectivity of posterior hippocampal regions to cortical DMN regions (Damoiseaux et al. 2016), as well as changes in intra-network connectivity of the DMN (Ng et al. 2016). In sum, both our study and previous work tentatively suggest that lower connectivity within the MTL_RSC-DMN might not be directly related to memory performance, but instead might indicate that stronger connectivity of the MTL_RSC-DMN to cortical regions outside this network explains individual variation in memory ability, depending on age. However, the dependency on age appears to be complex, necessitating more cross-sectional or even longitudinal research to disentangle the relation between MTL_RSC-DMN connectivity, age, and memory performance.

Previous studies have revealed better memory performance with increased connectivity between the posterior and anterior midline regions (Andrews-Hanna et al. 2007; Vidal-Piñeiro et al. 2014). Moreover, these midline core regions, together with the angular gyrus, have been implicated in memory retrieval (Vilberg and Rugg 2008; Sestieri et al. 2011, 2017; Kim 2015; Rugg and King 2017). Our finding of stronger connectivity between the main-DMN and the FPN associated with better memory performance may therefore be partly driven by these regions. While the DMN is assumed to be related to memory reconstruction and retrieval (Vincent et al. 2006; Kim 2015), the FPN is thought to underlie attention and cognitive control processes contributing to memory retrieval (Vincent et al. 2008). For example, the anterior inferior parietal lobe (Wagner et al. 2005; Cabeza et al. 2008; Ciaramelli et al. 2008; Vilberg and Rugg 2008; Sestieri et al. 2017) and the dorsolateral prefrontal cortex (Simons and Spiers 2003) support memory by mediating attentional control and monitoring processes. Furthermore, the FPN is thought to function as a flexible, adaptive system between internally focused processes of the DMN and more externally focused processes, such as bottom-up sensory processing and top-down attentional control (Dosenbach et al. 2007; Doucet et al. 2011; Cocchi et al. 2013; Cole et al. 2013). Our findings complement previous reports of task-based interactions between the DMN and FPN associated with mnemonic processing (Fornito et al. 2012; King et al. 2015; Kragel and Polyn 2015). In a recent study, King et al. (2015) revealed retrieval-related increases in connectivity between regions largely corresponding to the main-DMN and areas belonging to the FPN. Additionally, stronger connectivity between the DMN and the right FPN has been associated with more rapid recollection (Fornito et al. 2012). Resting-state interactions between the main-DMN and FPN may promote individual memory ability through dynamic interplay between processes directly related to memory recollection mediated by the main-DMN, and attention as well as memory control processes subserved by the FPN. Moreover, we speculate that enhanced interactions might enable better switching between internally focused memory processes and the processing of external cues. This could possibly result in better adaption to changing attentional demands that are required for adequate task performance.

In line with the suggested role of the main-DMN and FPN in memory, we showed increased activation during successful compared to unsuccessful retrieval during the delayed recall task in both networks. However, no significant differential activation in the MTL_RSC-DMN was observed during the task. Although the MTL_RSC-DMN showed generally elevated levels of activation during memory retrieval compared to baseline, the network did not differentiate between correct and incorrect trials. A reason for this could be that incorrect trials activated knowledge of the surrounding locations on the grid, or that the spatial associative and navigational aspects of the task required involvement of the MTL_RSC-DMN (Epstein 2008; Miller et al. 2014), irrespective of memory outcome. Future studies should focus on the effects of navigation in the regions of the MTL_RSC-DMN in a spatial associative memory task such as the one at hand.

In this study, we were interested in resting-state network dynamics in relation to individual variation in memory performance. To this aim, we focused on network-level interactions rather than separate regions, or region-to-region interactions. Therefore, we cannot make inferences about single regions that may drive within- or between-network interactions related to memory. Additionally, we targeted differences in individual memory ability by measuring memory performance on a cued recall, spatial associative task. However, it is difficult to draw conclusions specific to retrieval as this task is our only behavioral read-out. Differences in memory performance may therefore also reflect differences in memory encoding, consolidation or related attentional or memory search processes. A recent study of Sneve et al. (2017) targeted memory consolidation specifically. The authors showed that individuals with enhanced intrinsic connectivity between the hippocampus, DMN and FPN subnetworks, parietal and limbic subnetworks, had better memory performance $6.5 \mathrm{wk}$ after encoding. No significant association was found in participants whose memory was probed $1.5 \mathrm{~h}$ after consolidation, potentially reflecting long-term memory consolidation. Future studies investigating the role of intrinsic fluctuations in memory may consider 
including measures of encoding and consolidation to be able to draw more specific conclusions about the different memory processes. Moreover, future studies may consider investigating the extent to which our findings generalize over different forms of episodic memory. However, we used a typical episodic memory task, and given the importance of both the FPN and DMN in episodic memory and the previously reported finding of task-related connectivity changes between the FPN and DMN related to individual differences in episodic memory performance (King et al. 2015), we speculate that our findings can be generalized to other forms of episodic memory, at least in young adults. Furthermore, our model explained $51.2 \%$ of inter-individual variance in memory performance through network interactions within the MTL RSC-DMN and between the main-DMN and FPN. This amount of variance in memory accounted for by the interactions is specific to our statistical model. In reality, other factors such as structural brain connectivity and intelligence might contribute to individual differences in memory ability. Nevertheless, the large percentage of explained variance in our model does indicate an important role of resting-state functional connectivity within the MTL_RSC-DMN, and between the main-DMN and FPN in supporting individual memory ability. Moreover, control analyses revealed that network interactions between and within the auditory and visual network did not explain inter-individual differences in memory performance, suggesting that the network interactions within the MTL_RSC-DMN and between the main-DMN and FPN specifically contribute to individual memory ability. Finally, although it is likely that network interactions during resting-state reflect trait-like differences that are related to individual variation in memory performance (Geerligs et al. 2015; Touroutoglou et al. 2015), we cannot rule out the influence of state-like characteristics. Importantly, participants were aware that the resting-state measurement was followed by a recall task, and were possibly engaged in rehearsing the studied associations, which perhaps affected intrinsic brain connectivity.

To conclude, we found lower resting-state connectivity within the MTL_RSC-DMN network combined with stronger connectivity between the main-DMN and FPN explained differences in individual memory ability, as assessed by successful memory performance on a spatial associative retrieval task. Furthermore, exploratory whole-brain analysis revealed that stronger MTL connectivity with the left posterior parietal cortex was related to better memory performance, suggesting that lower intrinsic connectivity within the MTL_RSC-DMN may be indicative of a more functionally embedded MTL_RSC-DMN with neocortical regions. The functional importance of the main-DMN and FPN in memory was mirrored by changes in activation during the actual memory retrieval task, however, no retrieval-related activation differences were shown in the MTL_RSC-DMN.

\section{Materials and Methods}

\section{Subjects}

Data of 28 healthy males were acquired. Three participants were excluded because of technical failure of the cardiorespiratory recordings and one participant was excluded because he did not perform the task as instructed. This left 24 participants (age 19-29 yr, mean $22.69 \mathrm{yr}$ ) for data analyses. All participants were righthanded and none received medication, had any contraindication for MRI, a self-reported current disease or a past neurological, endocrine, psychiatric or substance use disorder. The participants took part in a randomized, double-blind, placebo-controlled, betweensubject study probing the effect of methylphenidate on memory (Wagner et al. 2017). Only male participants were included in this study to rule out interaction effects between methylphenidate and the menstrual cycle, as well as to avoid the inclusion of women who were in the early weeks of pregnancy. For the current study, only those who received placebo were included in the main analyses. Twenty-five participants who received methylphenidate were included in control analyses (see Supplemental Materials and Methods). The experimental protocol was reviewed and approved by the institutional review board (CMO Region ArnhemNijmegen, The Netherlands; registration number 2014/289). Participants received monetary compensation for participation.

\section{Procedure and associative memory task}

\section{General procedure}

Participants came to the laboratory on $2 \mathrm{~d}, 72 \mathrm{~h}$ apart (Fig. 1A). On the first day, participants performed the learning phase and the immediate recall test of an object-location association memory task on a computer in a behavioral laboratory (Fig. 1B,C). On the fourth day, participants returned to the center for a session in the MR scanner. Brain activity was measured using functional MRI during a resting-state period and subsequently while the participants performed the delayed recall test of the memory task.

\section{Resting-state}

To measure intrinsic brain activity, participants were scanned during a resting-state period of $11 \mathrm{~min}$ and were instructed to lie still and to look at a fixation cross. Eye-tracking was performed to ensure that they remained awake with their eyes open.

\section{Associative memory task}

On the first day, participants came to the behavioral laboratory to perform the associative memory task (Fig. 1, see also Wagner et al. 2017). Spatial associative memory tasks such as the task at hand tap into a core feature of episodic memory and have been used repeatedly in prior studies. They have been found to elicit robust activation in both the DMN and the FPN (Takashima et al. 2009; van Dongen et al. 2011, 2012 van Buuren et al. 2014; Wagner et al. 2016). In the current task, participants were instructed to memorize associations between pictures of everyday objects and their locations within an $8 \times 8$ grid presented on the computer screen, and to select the correct object-location association. First, participants practiced with the trackball (Kensington, Orbit Optical Trackball) used to select a location on the grid. After this practice session, the learning phase of the associative memory task began. At the start of this phase, all 64 objects were displayed at their correct screen locations within the grid for $1.5 \mathrm{~min}$. All objects were selected from the Hemera Photo-Objects database (Hemera Technologies Inc.) and were distinct and easy to name. The assignment of the objects to the 64 grid locations was randomized across subjects. After viewing the grid, the first trial started with the presentation of a cue (i.e., one of the objects) in a red frame below an empty grid (Fig. 1B). After $3 \mathrm{sec}$, the frame turned green and a black squared cursor appeared at one of the four sides of the grid. Participants had to select the location on the grid that was associated with the object by scrolling the black squared cursor toward that location and by pressing the left button on the trackball within a response period of 2 sec. After responding, feedback was presented on the screen for $3 \mathrm{sec}$ plus the remaining response period. That is, if the given response was incorrect, the cursor turned red and the object was displayed at the correct location. If the response was correct, the object was shown at that location. After presentation of all objects, a rest block of 30 sec was inserted during which a fixation cross was presented on the screen. The learning phase consisted of five cycles, in which every object was presented once. The order of trials was randomized across subjects and per cycle.

Approximately $5 \mathrm{~min}$ after the learning phase, the recall phase started (Fig. 1C). In this phase, each object was presented once and sequentially below the board in a red frame for $3 \mathrm{sec}$ as a cue. Similar to the learning phase, subjects had to select the correct location (but within $2.5 \mathrm{sec}$ ). However, after selection of the card, no feedback was provided. The cursor turned gray for the remaining response period, followed by a 2.5-7.5 sec (mean $5 \mathrm{sec}$ ) 
inter-trial interval, during which a fixation cross was presented. After every 16 trials, a short break of $30 \mathrm{sec}$ (fixation cross) was inserted. Again the order of trials was randomized across subjects.

Memory was tested once again after $72 \mathrm{~h}$ (mean $=72 \mathrm{~h}$; range $=70-73 \mathrm{~h}$ ). The task instructions were repeated and participants were positioned in the MR scanner, where they lay supine with the trackball placed on their abdomen (Kensington, Orbit Optical Trackball, in house adapted for MRI compatibility). They could view the task on the screen through a mirror mounted on the head coil. First, brain activity during resting-state was measured. Then, participants practiced with the trackball and performed the final recall test, which was identical to the immediate recall test on the first day. The presentation order of the trials was again randomized across participants.

\section{Behavioral data analysis}

In line with our previous study (Wagner et al. 2017), successful memory performance was defined as the number of object-location associations that were correctly retrieved at both the immediate and the delayed recall test. Incorrect retrieval was defined by the amount of incorrect responses on the delayed recall test, and trials without a response were regarded as misses. The task and number of learning cycles were piloted to acquire both a high level of correct responses and an adequate distribution of correct and incorrect trials required for functional MRI analyses. Associations that were correctly remembered at the delayed recall test but incorrectly remembered at the immediate recall test were sparse (mean $\pm \mathrm{SE}$ : $2.63 \pm 0.26$ associations) and excluded from behavioral data analyses.

\section{Cardiorespiratory measurements and calculation}

Heart beat and respiration were measured during scanning as these processes affect the blood oxygen level-dependent (BOLD) signal independent of neuronal activity (Glover et al. 2000; Wise et al. 2004) and were found to affect functional connectivity measures (Birn et al. 2006; Shmueli et al. 2007; van Buuren et al. 2009). Heartbeat was measured by a pulse oximeter affixed to the little finger of the left hand and respiration was measured with a respiration belt placed at the level of the abdomen. Data were recorded with a MR-compatible BrainAmp MR amplifier (BrainProducts), and recordings as well as storage were controlled using the Brain Vision Recorder (BrainProducts).

Raw physiology recordings were converted using FieldTrip software (Oostenveld et al. 2011), artifacts in the signal caused by radiofrequency pulses were removed and peaks in the heart beat signal were detected using in-house developed software programmed in MATLAB (MATLAB 2014, The MathWorks Inc.). Next, heart beat data were visually inspected and spurious peaks were removed. To correct the BOLD signal for confounds caused by the respiratory cycle and cardiac pulsatility, RETROICOR (Glover et al. 2000) was applied to model the relationship between the BOLD signal and the phase of the filtered respiratory and cardiac signal using fifth-order Fourier series. This resulted in 10 regressors modeling the respiratory phase and 10 regressors modeling the cardiac phase for each subject. In addition, time courses of heart rate frequency and respiration volume per unit time were calculated and shifted with 6,10 , and $12 \mathrm{sec}$, and -1 and $5 \mathrm{sec}$, respectively, to account for the variable delay between fluctuations in these processes and the BOLD signal (Birn et al. 2006; Shmueli et al. 2007; van Buuren et al. 2009). This yielded three regressors for heart rate frequency and two regressors modeling respiration volume per unit time, resulting in a total of 25 regressors for each subject modeling cardiorespiratory effects on the BOLD signal.

\section{Definition of regions of interest}

The coordinates of the regions constituting the DMN and FPN were based on current literature describing the brain's resting-state dynamics (Yeo et al. 2011), and fractionation within the DMN (Andrews-Hanna et al. 2010). ROIs were all defined by $8-\mathrm{mm}$ spheres centered on the selected coordinates. We included mir- rored coordinates in the right hemisphere for lateral regions $(x>$ $-10)$ as was done in a previous study on network connectivity (Vatansever et al. 2015), since we were uncertain about possible laterality effects (Bellana et al. 2016). After careful inspection of the DMN ROIs (Andrews-Hanna et al. 2010), we had to omit the "hippocampal formation" ROI, because of its location ventral to the hippocampus, and near the edge of the brain resulting in poor temporal signal-to-noise ratio (tSNR left hippocampal formation: mean $=47.75$, range $=38.2-58.47$; tSNR right hippocampal formation: mean $=43.88$, range $=35.48-53.30$ ), despite sufficient tSNR in the hippocampus overall as based on the LONI Probabilistic Brain Atlas (Shattuck et al. 2008), using a probability of 75\%) (tSNR left hippocampus: mean $=90.32$, range $=63.34-108.53$; tSNR right hippocampus: mean $=94.08$, range $=72.18-107.80$ ) . The parahippocampal ROI had better tSNR but was located at the posterior end of the MTL. The MTL region described in Yeo and colleagues (Yeo et al. 2011 and see Table 1 for coordinates), and the mirrored region in the right hemisphere, were positioned between, and partly overlapping with, the "hippocampal formation" and parahippocampal ROIs defined in the study of Andrews-Hanna et al. (2010) and had good tSNR for analysis (tSNR left MTL: mean=91.10, range=74.56-102.99; tSNR right $\mathrm{MTL}$ : mean=89.60, range= 72.47-104.18). Given its location and the higher tSNR values, we included this MTL region described in Yeo et al. (2011) instead of the "hippocampal formation" and parahippocampal region centered on the coordinates provided in the study of AndrewsHanna et al. (2010). This resulted in a total of 22 regions; 14 regions constituting the DMN and eight regions constituting the FPN (see Table 1).

\section{MRI data acquisition and preprocessing}

\section{Data acquisition}

Participants were scanned using a Siemens Magnetom Skyra 3 Tesla MR scanner equipped with a 32-channel-phased array head coil. A total of 316 BOLD-dependent scans were acquired during the resting-state period, and 364 scans were obtained during the recall test, both using a T2*-weighted gradient-echo, multiecho echo planar imaging (EPI) sequence (Poser et al. 2006) with the following parameters: repetition time $(\mathrm{TR})=2100 \mathrm{msec}$; echo time $(\mathrm{TE}) 1=$ $8.5 \mathrm{msec}$, TE2 $=19.3 \mathrm{msec}$, TE3 = $30 \mathrm{msec}, \mathrm{TE} 4=41 \mathrm{msec}$; flip angle $=90^{\circ}$; matrix size $=64 \times 64$; field of view $(\mathrm{FOV})=224 \times 224 \times$ 119 ; slice thickness $=3 \mathrm{~mm}$; slice gap $=0.51 \mathrm{~mm}$; 34 slices, acquired in ascending order.

Between the acquisition of the resting-state and task functional data, a T1-weighted structural image of the whole brain was made using a magnetization prepared, rapid-acquisition gradient echo (MPRAGE) sequence (parameters: $\mathrm{TR}=2300 \mathrm{~ms}$; $\mathrm{TE}=3.03$ $\mathrm{ms}$; flip angle $=8^{\circ}$; matrix size $=256 \times 256$; FOV $=192 \times 256 \times 256$; slice thickness $=1 \mathrm{~mm}$; 192 sagittal slices).

\section{Data preprocessing}

Raw functional multiecho data acquired during resting-state were combined using in-house built MATLAB software. This software applies motion correction on the first echo by estimating iterative rigid body realignment to minimize the residual sum of squares between the first echo of the first scan and all other scans. These estimated parameters were applied to all other echoes, thereby realigning all echoes to the first echo of the first scan. Then, the four echo images of each scan were combined into a single image per scan using the weighted sum of the four echo times. These combined images were further spatially preprocessed using SPM8 (http://www.fil.ion.ucl.ac.uk/spm). First, using mutual information optimization, the individual structural image was co-registered to the mean functional scan, obtained after realignment. Next, the structural scan was segmented and the normalization parameters were estimated using unified segmentation. Subsequently, these parameters were used to transform both the structural image and the functional scans into Montreal Neurological Institute (MNI) space, as defined by the SPM8 MNI T1 template (resampled voxel size of functional data $=3.5 \times 3.5 \times 3.5 \mathrm{~mm}$ ). Finally, the functional 
scans were smoothed using a 3D Gaussian filter (8-mm full width at half maximum).

Spatial preprocessing of the functional data obtained during the recall test largely followed the same procedure. However, to combine the echoes of these scans into single images, 32 scans acquired during a brief additional rest period before start of the task were used to determine the optimal weighting of echo-times for each voxel, by calculating the contrast-to-noise ratio for each echo per scan. These calculated optimal echo time weightings were then used to combine the four echo images of each scan into single images after motion correction. Spatial preprocessing steps using SPM8 were performed on the combined images as described above.

Smoothed and normalized resting-state fMRI data were submitted to a voxel-wise multiple linear regression analysis using MATLAB to correct for effects of motion, cardiorespiratory processes, and low-frequency drifts. This regression contained the 25 regressors modeling the cardiorespiratory processes and the six realignment parameters. Motion scrubbing was performed to remove scans displaying excessive head motion, by calculating the framewise displacement (FD, Power et al. 2012, mean \pm SE: 0.12 \pm $0.01 \mathrm{~mm})$. This yielded one regressor per outlier-scan $(\mathrm{FD}>0.3$ $\mathrm{mm}$, mean \pm SE: $10 \pm 1.84$ outlier-scans), with a 1 coding for the outlier-time point and 0 coding for all other time points. In addition, we included a regressor of the average partial global signal of the brain to remove non-regional specific fluctuations affecting connectivity measures. This regressor contained the average BOLD signal time course of resting-state networks of no interest. These networks were the visual, cerebellar, sensorimotor and auditory networks, determined by the 10 well-matched networks of the resting-state data from the 20-component analysis published by Smith et al. (2009). Any overlapping voxels of these networks with the ROIs of the current study were removed. We included this partial global signal instead of the global signal of the entire brain to reduce the chance of inducing spurious correlations, or removing signal of interest. Finally, we added a discrete cosine transform basis set to the regression to remove low-frequency fluctuations below the frequencies of interest $(>0.01 \mathrm{~Hz})$. No low-pass filtering was performed as recent studies suggest that fluctuations at higher frequencies may contribute to the signature of the time courses and removal of these frequencies may result in less reliable connectivity profiles (Shirer et al. 2015; Geerligs et al. 2017), or even artificial correlations (Davey et al. 2013). Residual data were used to calculate connectivity between the ROIs. Notably, to allow a maximum delay between heart rate frequency and the BOLD-signal fluctuations of $12 \mathrm{sec}$, the first six scans and corresponding regressors were removed, leaving 310 scans for data analysis.

\section{Functional MRI data analyses}

\section{Resting-state functional connectivity analyses}

First, for each subject and each ROI, the average BOLD signal time course was extracted from the residual resting-state data. Correlation coefficients (Pearson's $r$ ) between the average time courses of each ROI with all other ROIs were calculated and the correlations were converted to $\mathrm{F} z$. These values were then averaged across subjects, resulting in a $22 \times 22$ average connectivity matrix. Second, to define subnetworks within the DMN, and potentially within the FPN, and to identify regions that changed network alignment, we calculated the Euclidean distance between average pairwise connectivity values and performed a hierarchical cluster analysis (using MATLAB's average "linkage" function). The resulting clusters were visualized with a dendrogram using a threshold of $70 \%$ of the maximum linkage, corresponding to four clusters. Third, to verify if within-network connectivity was significantly higher than between-network connectivity, we calculated connectivity within and between the resulting networks. To avoid circularity, we performed this verification using resting-state data of an independent group of 25 participants (see also Supplemental Materials and Methods and the final paragraph of the section Results-Network connectivity is associated with memory performance).
Individual correlation coefficients within and between the networks were again transformed to $\mathrm{Fz}$ and effects were tested using paired-sample $t$-tests. As a fourth step, we assessed whether interactions within and between the networks, as identified by the hierarchical cluster analysis, predicted memory performance. Therefore, we submitted the individual within- and between-network connectivity values $(\mathrm{Fz})$ into a multiple regression analysis with successful memory performance (see above) as a dependent variable. We used a stepwise regression approach (with a probability of $F$ to enter of $P<0.05$ ), since we did not expect all within and between intrinsic network interactions to contribute to successful memory performance.

\section{Task-induced activation}

To verify the involvement of DMN and FPN subnetworks (defined by the hierarchical cluster analysis on the resting-state data) in memory retrieval, the preprocessed functional images of the recall task were submitted to a general linear model (GLM) regression analysis. In this analysis, we included two regressors of interest; (1) correct trials, associations that were correctly remembered at both the delayed test and the immediate recall test, and (2) incorrect trials, associations that were incorrectly remembered at the delayed recall test. For these regressors, a parametric modulation of reaction time was included to capture variance in the signal linearly related to reaction time and not explained by signal changes attributed to the regressor (correct or incorrect). A regressor of no interest was included to model misses and associations that were correctly remember at the delayed recall test but incorrectly remembered at the immediate recall test. All regressors were timelocked to the onset of the cue and modeled by convolving a box-car function of $5.5 \mathrm{sec}$ (cue presentation + maximum response period) with a canonical hemodynamic response function (Friston et al. 1995). To correct for head motion, the six realignment parameters were included in the design matrix as regressors of no interest. A high-pass filter was applied to the data to remove low-frequency fluctuations in the signal (cutoff $128 \mathrm{sec}$ ). Subsequently, contrast images were created for each subject, comparing correct and incorrect recall to baseline, and the percent signal change was extracted for the networks derived from the hierarchical cluster analysis on the preceding resting-state data. Significance of activation within the networks was tested using a repeated-measures ANOVA with network and memory (correct, incorrect) as within-subject factors.

\section{Acknowledgments}

The authors thank Leonore Bovy for her assistance with data acquisition. This work was supported by the European Research Council (ERC R0001075 to G.F.).

\section{References}

Andrews-Hanna JR, Snyder AZ, Vincent JL, Lustig C, Head D, Raichle ME, Buckner RL. 2007. Disruption of large-scale brain systems in advanced aging. Neuron 56: 924-935. doi:10.1016/j.neuron.2007.10.038

Andrews-Hanna JR, Reidler JS, Sepulcre J, Poulin R, Buckner RL. 2010. Functional-anatomic fractionation of the brain's default network. Neuron 65: 550-562. doi:10.1016/j.neuron.2010.02.005

Andrews-Hanna JR, Saxe R, Yarkoni T. 2014a. Contributions of episodic retrieval and mentalizing to autobiographical thought: evidence from functional neuroimaging, resting-state connectivity, and fMRI meta-analyses. Neuroimage 91: 324-335. doi:10.1016/j. neuroimage.2014.01.032

Andrews-Hanna JR, Smallwood J, Spreng RN. 2014b. The default network and self-generated thought: component processes, dynamic control, and clinical relevance. Ann N Y Acad Sci 1316: 29-52. doi:10.1111/ nyas. 12360

Arslan S, Ktena SI, Makropoulos A, Robinson EC, Rueckert D, Parisot S. 2018. Human brain mapping: a systematic comparison of parcellation methods for the human cerebral cortex. Neuroimage 170: 5-30. doi:10.1016/j.neuroimage.2017.04.014

Bellana B, Liu Z, Anderson JAE, Moscovitch M, Grady CL. 2016. Laterality effects in functional connectivity of the angular gyrus during rest and episodic retrieval. Neuropsychologia 80: 24-34. doi:10.1016/j. neuropsychologia.2015.11.004 
Benoit RG, Schacter DL. 2015. Specifying the core network supporting episodic simulation and episodic memory by activation likelihood estimation. Neuropsychologia 75: 450-457. doi:10.1016/j. neuropsychologia.2015.06.034

Birn RM, Diamond JB, Smith MA, Bandettini PA. 2006. Separating respiratory-variation-related fluctuations from neuronal-activity-related fluctuations in fMRI. Neuroimage 31: 1536-1548. doi:10.1016/j. neuroimage.2006.02.048

Borst JP, Anderson JR. 2013. Using model-based functional MRI to locate working memory updates and declarative memory retrievals in the fronto-parietal network. Proc Natl Acad Sci 110: 1628-1633. doi:10.1073/pnas.1221572110

Buckner RL, Andrews-Hanna JR, Schacter DL. 2008. The brain's default network: anatomy, function, and relevance to disease. Ann N Y Acad Sci 1124: 1 -38. doi:10.1196/annals.1440.011

Cabeza R, Ciaramelli E, Olson IR, Moscovitch M. 2008. The parietal cortex and episodic memory: an attentional account. Nat Rev Neurosci 9: 613625. doi:10.1038/nrn2459

Ciaramelli E, Grady CL, Moscovitch M. 2008. Top-down and bottom-up attention to memory: a hypothesis (AtoM) on the role of the posterior parietal cortex in memory retrieval. Neuropsychologia 46: 1828-1851. doi:10.1016/j.neuropsychologia.2008.03.022

Cocchi L, Zalesky A, Fornito A, Mattingley JB. 2013. Dynamic cooperation and competition between brain systems during cognitive control. Trends Cogn Sci 17: 493-501. doi:10.1016/j.tics.2013.08.006

Cole MW, Reynolds JR, Power JD, Repovs G, Anticevic A, Braver TS. 2013. Multi-task connectivity reveals flexible hubs for adaptive task control. Nat Neurosci 16: 1348-1355. doi:10.1038/nn.3470

Damoiseaux JS, Beckmann CF, Arigita EJ, Barkhof F, Scheltens P, Stam CJ, Smith SM, Rombouts SA. 2008. Reduced resting-state brain activity in the "default network" in normal aging. Cereb Cortex 18: 1856-1864. doi:10.1093/cercor/bhm207

Damoiseaux JS, Viviano RP, Yuan P, Raz N. 2016. Differential effect of age on posterior and anterior hippocampal functional connectivity. Neuroimage 133: 468-476. doi:10.1016/j.neuroimage.2016.03.047

Daselaar SM, Prince SE, Cabeza R. 2004. When less means more: deactivations during encoding that predict subsequent memory. Neuroimage 23: 921-927. doi:10.1016/j.neuroimage.2004.07.031

Daselaar SM, Prince SE, Dennis NA, Hayes SM, Kim H, Cabeza R. 2009. Posterior midline and ventral parietal activity is associated with retrieval success and encoding failure. Front Hum Neurosci 3: 13. doi:10.3389/ neuro.09.013.2009

Davey CE, Grayden DB, Egan GF, Johnston LA. 2013. Filtering induces correlation in fMRI resting state data. Neuroimage 64: 728-740. doi:10.1016/j.neuroimage.2012.08.022

Dosenbach NU, Fair DA, Miezin FM, Cohen AL, Wenger KK, Dosenbach RA Fox MD, Snyder AZ, Vincent JL, Raichle ME, et al. 2007. Distinct brain networks for adaptive and stable task control in humans. Proc Natl Acad Sci 104: 11073-11078. doi:10.1073/pnas.0704320104

Doucet G, Naveau M, Petit L, Delcroix N, Zago L, Crivello F, Jobard G, Tzourio-Mazoyer N, Mazoyer B, Mellet E, et al. 2011. Brain activity at rest: a multiscale hierarchical functional organization. J Neurophysiol 105: 2753-2763. doi:10.1152/jn.00895.2010

Epstein R. 2008. Parahippocampal and retrosplenial contributions to human spatial navigation. Trends Cogn Sci 12: 388-396. doi:10.1016/j. tics.2008.07.004

Fjell AM, Sneve MH, Storsve AB, Grydeland H, Yendiki A, Walhovd KB. 2016. Brain events underlying episodic memory changes in aging: a longitudinal investigation of structural and functional connectivity. Cereb Cortex 26: 1272-1286. doi:10.1093/cercor/bhv102

Fornito A, Harrison BJ, Zalesky A, Simons JS. 2012. Competitive and cooperative dynamics of large-scale brain functional networks supporting recollection. Proc Natl Acad Sci 109: 12788-12793. doi:10.1073/pnas.1204185109

Fox MD, Snyder AZ, Vincent JL, Corbetta M, Van Essen DC, Raichle ME. 2005. The human brain is intrinsically organized into dynamic, anticorrelated functional networks. Proc Natl Acad Sci 102: 9673-9678. doi:10.1073/pnas.0504136102

Friston KJ, Frith CD, Turner R, Frackowiak RSJ. 1995. Characterizing evoked hemodynamics with fMRI. Neuroimage 2: 157-165. doi:10.1006/ nimg.1995.1018

Geerligs L, Rubinov M, Cam-CAN, Henson RN. 2015. State and trait components of functional connectivity: individual differences vary with mental state. J Neurosci 35: 13949-13961. doi:10.1523/ JNEUROSCI.1324-15.2015

Geerligs L, Tsvetanov KA, Cam-CAN, Henson RN. 2017. Challenges in measuring individual differences in functional connectivity using fMRI: the case of healthy aging. Hum Brain Mapp 38: 4125-4156. doi:10.1002/ hbm. 23653

Gerraty RT, Davidow JY, Wimmer GE, Kahn I, Shohamy D. 2014. Transfer of learning relates to intrinsic connectivity between hippocampus, ventromedial prefrontal cortex, and large-scale networks. J Neurosci 34: 11297-11303. doi:10.1523/JNEUROSCI.0185-14.2014

Glover GH, Li TQ, Ress D. 2000. Image-based method for retrospective correction of physiological motion effects in fMRI: RETROICOR. Magn Reson Med 44: 162-167. doi:10.1002/1522-2594(200007)44:1\&lt;162:: AID-MRM23\&gt;3.0.CO;2-E

Greicius MD, Krasnow B, Reiss AL, Menon V. 2003. Functional connectivity in the resting brain: a network analysis of the default mode hypothesis. Proc Natl Acad Sci 100: 253-258. doi:10.1073/pnas.0135058100

Gusnard DA, Raichle ME. 2001. Searching for a baseline: functional imaging and the resting human brain. Nat Rev Neurosci 2: 685-694. doi:10.1038 35094500

Huijbers W, Vannini P, Sperling RA, Pennartz CM, Cabeza R, Daselaar SM. 2012. Explaining the encoding/retrieval flip: memory-related deactivations and activations in the posteromedial cortex. Neuropsychologia 50: 3764-3774. doi:10.1016/j. neuropsychologia.2012.08.021

Kaboodvand N, Bäckman L, Nyberg L, Salami A. 2018. The retrosplenial cortex: a memory gateway between the cortical default mode network and the medial temporal lobe. Hum Brain Mapp 39: 2020-2034. doi: $10.1002 / \mathrm{hbm} .23983$

Kim H. 2015. Encoding and retrieval along the long axis of the hippocampus and their relationships with dorsal attention and default mode networks: the HERNET model. Hippocampus 25: 500-510. doi:10.1002/hipo.22387

King DR, de Chastelaine M, Elward RL, Wang TH, Rugg MD. 2015. Recollection-related increases in functional connectivity predict individual differences in memory accuracy. J Neurosci 35: 1763-1772. doi:10.1523/JNEUROSCI.3219-14.2015

Kragel JE, Polyn SM. 2015. Functional interactions between large-scale networks during memory search. Cereb Cortex 25: 667-679. doi:10.1093/cercor/bht 258

Kumaran D, Summerfield JJ, Hassabis D, Maguire EA. 2009. Tracking the emergence of conceptual knowledge during human decision making. Neuron 63: 889-901. doi:10.1016/j.neuron.2009.07.030

La Joie R, Landeau B, Perrotin A, Bejanin A, Egret S, Pélerin A, Mézenge F, Belliard S, de La Sayette V, Eustache F, et al. 2014. Intrinsic connectivity identifies the hippocampus as a main crossroad between Alzheimer's and semantic dementia-targeted networks. Neuron 81: 1417-1428. doi:10.1016/j.neuron.2014.01.026

Markett S, Reuter M, Montag C, Voigt G, Lachmann B, Rudorf S, Elger CE, Weber B. 2014. Assessing the function of the fronto-parietal attention network: insights from resting-state fMRI and the attentional network test. Hum Brain Mapp 35: 1700-1709. doi:10.1002/hbm.22285

Meyer ML, Davachi L, Ochsner KN, Lieberman D. 2018. Evidence that default network connectivity during rest consolidates social information. Cereb Cortex doi:10.1093/cercor/bhy071

Miller AMP, Vedder LC, Law LM, Smith DM. 2014. Cues, context, and long-term memory: the role of the retrosplenial cortex in spatial cognition. Front Hum Neurosci 8: 586. doi:10.3389/fnhum.2014.00586

Ng KK, Lo JC, Lim JKW, Chee MWL, Zhou J. 2016. Reduced functional segregation between the default mode network and the executive control network in healthy older adults: a longitudinal study. Neuroimage 133: 321-330. doi:10.1016/j.neuroimage.2016.03.029

Oostenveld R, Fries P, Maris E, Schoffelen JM. 2011. FieldTrip: open source software for advanced analysis of MEG, EEG, and invasive electrophysiological data. Comput Intell Neurosci 2011: 156869. doi:10.1155/2011/156869

Poser BA, Versluis MJ, Hoogduin JM, Norris DG. 2006. BOLD contrast sensitivity enhancement and artifact reduction with multiecho EPI: parallel-acquired inhomogeneity-desensitized fMRI. Magn Reson Med 55: $1227-1235$. doi: $10.1002 / \mathrm{mrm} .20900$

Power JD, Barnes KA, Snyder AZ, Schlaggar BL, Petersen SE. 2012. Spurious but systematic correlations in functional connectivity MRI networks arise from subject motion. Neuroimage 59: 2142-2154. doi:10.1016/j. neuroimage.2011.10.018

Raichle ME, MacLeod AM, Snyder AZ, Powers WJ, Gusnard DA, Shulman GL. 2001. A default mode of brain function. Proc Natl Acad Sci 98: 676-682. doi:10.1073/pnas.98.2.676

Rugg MD, King DR. 2017. Ventral lateral parietal cortex and episodic memory retrieval. Cortex 107: 238-250. doi:10.1016/j.cortex. 2017.07.012

Salami A, Pudas S, Nyberg L. 2014. Elevated hippocampal resting-state connectivity underlies deficient neurocognitive function in aging. Proc Natl Acad Sci 111: 17654-17659. doi:10.1073/pnas.1410233111

Sestieri C, Corbetta M, Romani GL, Shulman GL. 2011. Episodic memory retrieval, parietal cortex, and the default mode network: functional and topographic analyses. J Neurosci 31: 4407-4420. doi:10.1523/ JNEUROSCI.3335-10.2011

Sestieri C, Shulman GL, Corbetta M. 2017. The contribution of the human posterior parietal cortex to episodic memory. Nat Rev Neurosci 18: 183192. doi:10.1038/nrn.2017.6 
Shattuck DW, Mirza M, Adisetiyo V, Hojatkashani C, Salamon G, Narr KL, Poldrack RA, Bilder RM, Toga AW. 2008. Construction of a 3D probabilistic atlas of human cortical structures. Neuroimage 39: 10641080. doi:10.1016/j.neuroimage.2007.09.031

Sheldon S, Farb N, Palombo DJ, Levine B. 2016. Intrinsic medial temporal lobe connectivity relates to individual differences in episodic autobiographical remembering. Cortex 74: 206-216. doi:10.1016/j. cortex.2015.11.005

Shirer WR, Jiang H, Price CM, Ng B, Greicius MD. 2015. Optimization of rs-fMRI pre-processing for enhanced signal-noise separation, test-retest reliability, and group discrimination. Neuroimage 117: 67-79. doi: 10.1016/j.neuroimage.2015.05.015

Shmueli K, van Gelderen P, de Zwart JA, Horovitz SG, Fukunaga M, Jansma JM, Duyn JH. 2007. Low-frequency fluctuations in the cardiac rate as a source of variance in the resting-state fMRI BOLD signal. Neuroimage 38: 306-320. doi:10.1016/j.neuroimage.2007.07.037

Shulman GL, Fiez JA, Corbetta M, Buckner RL, Miezin FM, Raichle M, Petersen SE. 1997. Common blood flow changes across visual tasks: II. Decreases in cerebral cortex. J Cogn Neurosci 9: 648-663. doi:10.1162/ jocn.1997.9.5.648

Simons JS, Spiers HJ. 2003. Prefrontal and medial temporal lobe interactions in long-term memory. Nat Rev Neurosci 4: 637-648. doi:10.1038/ nrn1178

Smith SM, Fox PT, Miller KL, Glahn DC, Fox PM, Mackay CE, Filippini N, Watkins KE, Toro R, Laird AR, et al. 2009. Correspondence of the brain's functional architecture during activation and rest. Proc Natl Acad Sci 106: $13040-13045$. doi:10.1073/pnas.0905267106

Sneve MH, Grydeland H, Amlien IK, Langnes E, Walhovd KB, Fjell AM. 2017. Decoupling of large-scale brain networks supports the consolidation of durable episodic memories. Neuroimage 153: 336-345. doi:10.1016/j.neuroimage.2016.05.048

Spreng RN, Stevens WD, Chamberlain JP, Gilmore AW, Schacter DL. 2010. Default network activity, coupled with the frontoparietal control network, supports goal-directed cognition. Neuroimage 53: 303-317. doi:10.1016/j.neuroimage.2010.06.016

Spreng RN, Sepulcre J, Turner GR, Stevens WD, Schacter DL. 2013. Intrinsic architecture underlying the relations among the default, dorsal attention, and frontoparietal control networks of the human brain. $J$ Cogn Neurosci 25: 74-86. doi:10.1162/jocn_a_00281

Spreng RN, Lockrow AW, DuPre E, Setton R, Spreng KAP, Turner GR. 2018. Semanticized autobiographical memory and the default-executive coupling hypothesis of aging. Neuropsychologia 110: $37-43$. doi:10.1016/j.neuropsychologia.2017.06.009

Staffaroni AM, Brown JA, Casaletto KB, Elahi FM, Deng J, Neuhaus J, Cobigo Y, Mumford PS, Walters S, Saloner R, et al. 2018. The longitudinal trajectory of default mode network connectivity in healthy older adults varies as a function of processing speed. J Neurosci 38: 28092817. doi:10.1523/JNEUROSCI.3067-17.2018

Takashima A, Nieuwenhuis IL, Jensen O, Talamini LM, Rijpkema M, Fernández G. 2009. Shift from hippocampal to neocortical centered retrieval network with consolidation. J Neurosci 29: 10087-10093.

Touroutoglou A, Andreano JM, Barrett LF, Dickerson BC. 2015. Brain network connectivity-behavioral relationships exhibit trait-like properties: evidence from hippocampal connectivity and memory. Hippocampus 25: 1591-1598. doi:10.1002/hipo.22480

van Buuren M, Gladwin TE, Zandbelt BB, van den Heuvel M, Ramsey NF, Kahn RS, Vink M. 2009. Cardiorespiratory effects on default-mode network activity as measured with fMRI. Hum Brain Mapp 30: 3031 3042. doi:10.1002/hbm.20729 van Buuren M, Gladwin TE, Zandbelt BB, Kahn RS, Vink M. 2010. Reduced functional coupling in the default-mode network during self-referential processing. Hum Brain Mapp 31: 1117-1127. doi:10.1002/hbm.20920

van Buuren M, Kroes MCW, Wagner IC, Genzel L, Morris RGM, Fernández G. 2014. Initial Investigation of the effects of an experimentally learned schema on spatial associative memory in humans. J Neurosci 34: 16662-16670.

van Dongen EV, Takashima A, Barth M, Fernández G. 2011. Functional connectivity during light sleep is correlated with memory performance for face-location associations. Neuroimage 57: 262-270.

van Dongen EV, Thielen JW, Takashima A, Barth M, Fernández G. 2012. Sleep supports selective retention of associative memories based on relevance for future utilization. PLoS One 7: e43426.

Vatansever D, Menon DK, Manktelow AE, Sahakian BJ, Stamatakis EA. 2015. Default mode network connectivity during task execution. Neuroimage 122: 96-104. doi:10.1016/j.neuroimage.2015.07.053

Vidal-Piñeiro D, Valls-Pedret C, Fernández-Cabello S, Arenaza-Urquijo EM, Sala-Llonch R, Solana E, Bargalló N, Junqué C, Ros E, Bartrés-Faz D. 2014. Decreased default mode network connectivity correlates with age-associated structural and cognitive changes. Front Aging Neurosci 6: 256. doi:10.3389/fnagi.2014.00256

Vilberg KL, Rugg MD. 2008. Memory retrieval and the parietal cortex: a review of evidence from a dual-process perspective. Neuropsychologia 46: 1787-1799. doi:10.1016/j.neuropsychologia.2008.01.004

Vincent JL, Snyder AZ, Fox MD, Shannon BJ, Andrews JR, Raichle ME, Buckner RL. 2006. Coherent spontaneous activity identifies a hippocampal-parietal memory network. J Neurophysiol 96: 3517-3531. doi:10.1152/jn.00048.2006

Vincent JL, Kahn I, Snyder AZ, Raichle ME, Buckner RL. 2008. Evidence for a frontoparietal control system revealed by intrinsic functional connectivity. J Neurophysiol 100: 3328-3342. doi:10.1152/jn. 90355.2008

Wagner AD, Shannon BJ, Kahn I, Buckner RL. 2005. Parietal lobe contributions to episodic memory retrieval. Trends Cogn Sci 9: 445-453. doi:10.1016/j.tics.2005.07.001

Wagner IC, van Buuren M, Bovy L, Fernández G. 2016. Parallel engagement of regions associated with encoding and later retrieval forms durable memories. J Neurosci 36: 7985-7995.

Wagner IC, van Buuren M, Bovy L, Morris RG, Fernández G. 2017. Methylphenidate during early consolidation affects long-term associative memory retrieval depending on baseline catecholamines. Psychopharmacology (Berl) 234: 657-669. doi:10.1007/s00213016-4502-8

Wang L, LaViolette P, O'Keefe K, Putcha D, Bakkour A, Van Dijk KRA, Pihlajamäki M, Dickerson BC, Sperling RA. 2010. Intrinsic connectivity between the hippocampus and posteromedial cortex predicts memory performance in cognitively intact older individuals. Neuroimage 51: 910-917. doi:10.1016/j.neuroimage.2010.02.046

Wise RG, Ide K, Poulin MJ, Tracey I. 2004. Resting fluctuations in arterial carbon dioxide induce significant low frequency variations in BOLD signal. Neuroimage 21: 1652-1664. doi:10.1016/j.neuroimage. 2003.11.025

Yeo BT, Krienen FM, Sepulcre J, Sabuncu MR, Lashkari D, Hollinshead M, Roffman JL, Smoller JW, Zöllei L, Polimeni JR, et al. 2011. The organization of the human cerebral cortex estimated by intrinsic functional connectivity. J Neurophysiol 106: 1125-1165. doi:10.1152/ jn.00338.2011

Received July 10, 2018; accepted in revised form November 17, 2018. 


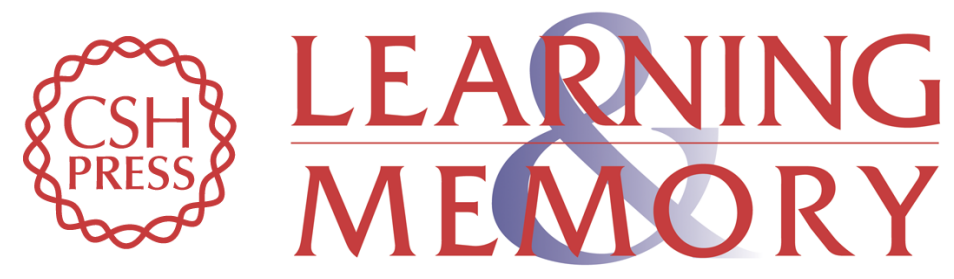

\section{Functional network interactions at rest underlie individual differences in memory ability}

Mariët van Buuren, Isabella C. Wagner and Guillén Fernández

Learn. Mem. 2019, 26:

Access the most recent version at doi:10.1101/Im.048199.118

\section{Supplemental http://learnmem.cshlp.org/content/suppl/2018/12/10/26.1.9.DC1 Material}

References This article cites 81 articles, 16 of which can be accessed free at: http://learnmem.cshlp.org/content/26/1/9.full.html\#ref-list-1

Creative This article is distributed exclusively by Cold Spring Harbor Laboratory Press for the Commons first 12 months after the full-issue publication date (see

License http://learnmem.cshlp.org/site/misc/terms.xhtml). After 12 months, it is available under a Creative Commons License (Attribution-NonCommercial 4.0 International), as described at http://creativecommons.org/licenses/by-nc/4.0/.

Email Alerting Receive free email alerts when new articles cite this article - sign up in the box at the Service top right corner of the article or click here. 